\title{
Análise de custos da assistência à saúde aos portadores de diabetes melito e hipertensão arterial em uma unidade de saúde pública de referência em Recife - Brasil
}

\author{
Analysis of health care costs of patients with diabetes mellitus and \\ hypertension in a public health reference unit in Recife - Brazil
}

Michelly Geórgia da Silva Marinho', Eduarda Ângela Pessoa Cesse', Adriana Falangola Benjamin Bezerra², Islândia Maria Carvalho de Sousa', Annick Fontbonne ${ }^{3}$, Eduardo Freese de Carvalho'

1 Departamento de Saúde Coletiva, Centro de Pesquisas Aggeu Magalhães, Fundação Oswaldo Cruz, Recife, PE, Brasil 2 Departamento de Medicina Social, Universidade Federal de Pernambuco (UFPE) Recife, PE, Brasil

${ }^{3}$ UMR 204 NutriPAss, IRD/ UM1/UM2/SupAgro - Institut de Recherche pour le Développement, Montpellier, França
Correspondência para: Michelly Geórgia da Silva Marinho Rua Mário Campelo, 201/406 50741-430 - Recife, PE, Brasil michelly_marinho@yahoo.com.br

Recebido em 8/Dez/2010 Aceito em 7/Ago/2011

\section{RESUMO}

Objetivo: Analisar os custos para a assistência à saúde de portadores de diabetes melito e hipertensão arterial e estimar o custo de procedimentos ambulatoriais de média complexidade comparando-os com os valores da tabela de reembolso do Sistema Único de Saúde (SUS). Materiais e métodos: Foram analisados os custos diretos sanitários, em unidade pública de referência em Recife/PE no ano de 2007. Para o levantamento e alocação dos custos, utilizaram-se as técnicas de custeio por absorção e de rateio. Resultados: Os custos diretos e o valor reembolsado pelo SUS totalizaram $\mathrm{R} \$ 4.855 .291,82$ e $\mathrm{R} \$ 2.118 .893,56$, respectivamente. Os grupos de despesas que apresentaram maiores custos foram: medicamentos $\mathrm{R} \$ 1.762 .424,42$ (36,3\%), serviços de terceiros $R \$ 996.637,82(20,5 \%)$ e pessoal $R \$ 978.096,10(20,1 \%)$. Todos os procedimentos apresentaram maior custo estimado que os valores pagos pela tabela SUS. Conclusões: Os medicamentos representaram os maiores custos para assistência e identificou-se diferença considerável entre os custos estimados e os valores reembolsados pelo SUS. Arq Bras Endocrinol Metab. 2011;55(6):406-11

\section{Descritores}

Diabetes melito; hipertensão arterial; custos e análise de custo; custos diretos de serviços; SUS

\section{ABSTRACT}

Objective: To analyze health care costs of patients with diabetes mellitus and hypertension, and to estimate the cost of medium complexity outpatient procedures, compared with the standard reimbursement values used in Brazil. Materials and methods: We analyzed direct health costs in a public health reference unit in Recife/PE, in 2007. Costs were determined and allocated using the techniques of absorption costing and apportionment. Results: Direct costs and the amount reimbursed by the SUS totaled $\mathrm{R} \$ 4,855,291.82$ and $\mathrm{R} \$ 2.118 .893,56$, respectively. The greatest groups of expenditure were medications, with $\mathrm{R} \$ 1,762,424.42$ (36.3\%), outsourced services, with $\mathrm{R} \$ 996,637.82$ (20.5\%); and personnel, with $\mathrm{R} \$ 978,096.10$ (20.1\%). All procedures had higher estimated costs than what is reimbursed by the SUS. Conclusions: Drugs were associated with the highest health care costs, a considerable difference was observed between estimated costs and the amount reimbursed by the SUS. Arq Bras Endocrinol Metab. 2011;55(6):406-11

Keywords

Diabetes mellitus; hypertension; costs and cost analysis; direct service costs; Unified Health Care System 


\section{INTRODUÇÃO}

$\mathrm{O}$

perfil epidemiológico brasileiro vem se modificando desde os anos 1950 e, neste cenário complexo, as doenças crônicas não transmissíveis (DCNT) atualmente assumem papel de destaque entre as principais causas de morbimortalidade $(1,2)$.

Esse fato leva à necessidade de reforço das ações de prevenção, diagnóstico precoce e tratamento para o controle das DCNT, em particular, o diabetes melito e a hipertensão arterial que são fatores de risco para outros agravos que acarretam altos custos, tanto do ponto de vista social quanto econômico (3-7).

A Federação Internacional de Diabetes estima que em 2010 os custos globais com a doença foram de US\$ 376,0 bilhões (8). Estudos de estimativa do custo anual associado à atenção aos portadores de diabetes melito nos Estados Unidos e na América Latina revelaram que os custos diretos e indiretos totalizaram US\$ 102,5 e US\$ 94,3 bilhões, respectivamente $(5,9)$. Análises de custo do tratamento para o diabetes em países latino-americanos demonstraram a significante carga econômica da doença para os sistemas de saúde e sociedade (10-12).

No Brasil, o custo anual estimado para o tratamento da hipertensão arterial no sistema público de saúde foi de US\$ 398,9 milhões e representou 1,43\% dos gastos totais do SUS (7). Estudos sobre custos hospitalares e com medicamentos por algumas doenças crônicas vêm demonstrando o impacto econômico dessas doenças para o SUS, bem como que o diabetes e a hipertensão são potenciais preditores clínicos para o agravamento dos casos, aumento do tempo de internação e apresentam consequentemente associação positiva com maior custo de tratamento (13-15).

Em países com as características do Brasil, onde os recursos para o setor saúde são escassos, torna-se essencial a eficiência na provisão dos serviços. No âmbito da economia da saúde, estudos como este têm gerado conhecimentos aplicáveis à realidade dos serviços, auxiliando na alocação de recursos e na determinação das prioridades da gestão em saúde $(16,17)$.

Este estudo analisou os custos para a assistência à saúde de portadores de diabetes melito e hipertensão arterial e estimou o custo de procedimentos ambulatoriais de média complexidade comparando-os com os valores da tabela de reembolso do SUS.

\section{MATERIAIS E MÉTODOS}

Realizou-se um estudo de análise de custos da provisão para a assistência à saúde em unidade de referên- cia de média complexidade localizada em Recife, sob gestão municipal e financiada com recursos do Sistema Único de Saúde e da Prefeitura da Cidade do Recife. A unidade é referência para todo o município com uma média anual de mais de 250 mil procedimentos, atende hipertensos e diabéticos nas especialidades de cardiologia, endocrinologia, enfermagem, nutrição, odontologia, oftalmologia e psicologia.

A análise abordou os custos do serviço sob a perspectiva do gestor público, no ano de 2007. Inicialmente, analisou-se a oferta dos serviços da Unidade de Referência e os recursos empregados para seu funcionamento e posteriormente relacionaram-se os resultados dessa oferta aos custos, de forma a estimar o custo médio de procedimentos ambulatoriais selecionados, comparando os valores estimados aos valores pagos pelo SUS.

Para o levantamento da produção ambulatorial e dos valores reembolsados pelo SUS, utilizaram-se os arquivos do banco de dados do Sistema de Informações Ambulatoriais (SIA/SUS) (18).

O estudo adotou para análise a classificação dos custos nos serviços de saúde elegendo a categoria dos custos diretos sanitários que são referentes aos custos dos funcionários, suprimentos e material de consumo, serviços de terceiros, instalações físicas, medicamentos, exames e serviços ambulatoriais $(16,17)$. Para a contabilização dos custos, utilizou-se a técnica de custeio por absorção que consiste na apropriação integral de todos os custos (17). Os valores em reais dos custos diretos foram apurados por meio dos dados do Sistema de Informação de Dispensação de Medicamentos e do Centro de Custos da Secretaria Municipal de Saúde fornecidos pela Prefeitura da Cidade do Recife.

Após a identificação dos custos, esses foram alocados de acordo com a divisão proposta por Falk (19). Segundo o autor, entre os tipos de custos que devem ser identificados estão: de pessoal, relativo aos salários dos profissionais de saúde; de capital, referente à aquisição e à manutenção de bens imóveis; e de despesas gerais (overhead), que são os custos para manter a estrutura física da unidade de saúde em funcionamento.

Em função da inexistência de um centro de custos produtivos no serviço de referência, para a estimativa do custo médio dos procedimentos foi desenvolvida a estratégia de criação de três categorias a partir dos custos diretos sanitários: Custo de funcionamento - constituído pelo somatório dos custos gerais (material de consumo, serviço de terceiros, serviços básicos) e dos custos de capital (Tabela 2); Custo de funcionamento por procedimento, no qual o Custo de funcionamento foi 
rateado proporcionalmente ao quantitativo produzido do procedimento; Custo de pessoal por procedimento cujo cálculo se faz pela soma dos valores referentes ao salário anual dos profissionais envolvidos na realização do procedimento rateado pelo número de horas dedicadas ao ambulatório e à realização de exames. A seleção dos procedimentos para a estimativa do custo médio unitário foi realizada de acordo com a possibilidade de aferir o Custo de pessoal por procedimento.

O estudo recebeu aprovação do Comitê de Ética e Pesquisa do Centro de Pesquisas Aggeu Magalhães (CEP/CPqAM), sob o registro de número 132/08.

\section{RESULTADOS}

Os procedimentos executados na unidade totalizaram 239.263/ano, e o valor reembolsado pelo SUS, por meio do Incentivo à Média e Alta Complexidade (MAC), gerou uma receita no valor de R\$ 2.118.893,56. Os procedimentos de finalidade diagnóstica representaram $57 \%$ do total da produção ambulatorial da unidade e $45 \%$ do valor pago pelo SUS. A oferta de consultas ambulatoriais especializadas de cardiologia, endocrinologia, enfermagem, nutrição, odontologia e oftalmologia representou aproximadamente $35 \%$ do total da produção. As cirurgias ambulatoriais representaram apenas $4 \%$ da produção e corresponderam a $18 \%$ do valor em Reais, com destaque para as cirurgias oftalmológicas, que corresponderam a $13 \%$ do total pago pelo SUS (Tabela 1).

$\mathrm{Na}$ tabela 2, os custos diretos sanitários da Unidade de Referência totalizaram R $\$ 4.855 .291,82$, sendo os medicamentos para doenças cardiovasculares responsáveis por $24,6 \%$ destes. Os grupos de despesas que apresentaram os maiores custos foram: medicamentos R\$ $1.762 .424,42(36,3 \%)$, serviços de terceiros R\$ 996.637,82 (20,5\%) e pessoal R\$ 978.096,10 (20,1\%).

A relação da receita (valor reembolsado pelo SUS, MAC - R\$ 2.118.893,56) e das despesas (gastos diretos sanitários, excluindo-se os valores dos medicamentos - R\$ 3.092867,40), resultou no saldo anual negativo de R\$ 973.973,84 para a Unidade de referência.

Em relação ao custo médio de procedimentos, as consultas especializadas e os exames de ecocardiografia e o teste ergométrico apresentaram equilíbrio quanto à composição dos custos totais e, de acordo com a metodologia adotada, os custos com pessoal foram superiores aos de funcionamento, exceto para o exame de eletrocardiograma no qual o custo de pessoal foi apenas $19,56 \%$ da composição do custo total (Tabela 3 ).
Ao comparar o custo estimado dos procedimentos realizados na unidade de referência aos valores da tabela SUS, observou-se que todos os procedimentos apresentaram custo médio maior que os valores em Reais reembolsados pelo sistema de saúde. O exame de eletrocardiograma e a consulta de nutrição apresentaram as maiores diferenças $+83 \% \mathrm{e}+197 \%$, respectivamente (Tabela 4).

Tabela 1. Número e valores pagos em reais dos procedimentos realizados na unidade de referência em 1 mês, 1 ano

\begin{tabular}{|c|c|c|c|c|}
\hline Procedimentos & $\mathbf{N}$ & $\%$ Proc & Valor (R\$) & $\begin{array}{c}\% \\
\text { (R\$) }\end{array}$ \\
\hline \multicolumn{5}{|c|}{ Grupo 1 - Ações de promoção e prevenção em saúde } \\
\hline $\begin{array}{l}\text { Atividades educativas em } \\
\text { grupo e individual }{ }^{\star}\end{array}$ & 1.947 & 0,8 & 0 & 0 \\
\hline Saúde bucal* ${ }^{*}$ & 1.046 & 0,4 & 0 & 0 \\
\hline Subtotal & 2.993 & 1,2 & 0 & 0 \\
\hline \multicolumn{5}{|c|}{ Grupo 2 - Procedimentos com finalidade diagnóstica } \\
\hline $\begin{array}{l}\text { Exames odontológicos } \\
\text { (radiografia) }\end{array}$ & 37 & 0,02 & 64,75 & 0,003 \\
\hline Exames cardiológicos & 21.112 & 8,8 & $223.852,40$ & 10,6 \\
\hline Exames oftalmológicos & 101.248 & 42,4 & $724.982,09$ & 34,2 \\
\hline $\begin{array}{l}\text { Exames endocrinológicos } \\
\text { (glicemia) }{ }^{\star}\end{array}$ & 13.739 & 5,7 & 0 & 0 \\
\hline Subtotal & 136.136 & 57,0 & $948.899,23$ & 44,8 \\
\hline \multicolumn{5}{|c|}{ Grupo 3 - Procedimentos clínicos } \\
\hline Aferição de pressão arterial* & 662 & 0,3 & 0 & 0 \\
\hline $\begin{array}{l}\text { Atendimento em serviço } \\
\text { social }\end{array}$ & 3.330 & 1,4 & $19.980,00$ & 0,9 \\
\hline Consulta em cardiologia & 16.710 & 7,0 & $167.100,00$ & 7,9 \\
\hline Consulta em endocrinologia & 17.598 & 7,4 & $175.980,00$ & 8,3 \\
\hline Consulta em enfermagem & 7.379 & 3,1 & $44.274,00$ & 2,1 \\
\hline Consulta em nutrição & 1.504 & 0,6 & $9.024,00$ & 0,4 \\
\hline $\begin{array}{l}\text { Consulta em odontologia } \\
\left(1^{\mathrm{a}} \text { consulta }\right)^{\star}\end{array}$ & 1.908 & 0,8 & 0 & 0 \\
\hline Consulta em oftalmologia & 35.975 & 15,1 & $359.750,00$ & 17,0 \\
\hline Consulta em psicologia & 1.017 & 0,4 & $6.102,00$ & 0,3 \\
\hline $\begin{array}{l}\text { Terapias em grupo e } \\
\text { individual }\end{array}$ & 1.784 & 0,7 & $6.339,76$ & 0,3 \\
\hline $\begin{array}{l}\text { Procedimentos } \\
\text { odontológicos }\end{array}$ & 2.401 & 1,0 & $2.843,25$ & 0,1 \\
\hline Subtotal & 90.268 & 37,8 & $791.393,01$ & 37,3 \\
\hline \multicolumn{5}{|c|}{ Grupo 4 - Procedimentos cirúrgicos } \\
\hline Cirurgias odontológicas & 7.805 & 3,3 & $106.250,15$ & 5,0 \\
\hline Cirurgias oftalmológicas & 1.824 & 0,7 & $272.351,17$ & 12,9 \\
\hline Subtotal & 9.629 & 4,0 & $378.601,32$ & 17,9 \\
\hline Total & 239.026 & 100 & $2.118 .893,56$ & 100 \\
\hline
\end{tabular}

* Procedimentos que não são reembolsados pela tabela SUS. Eles são financiados pelos recursos per capita do Piso da Atenção Básica (PAB) de repasse fundo a fundo do Ministério da Saúde para o Fundo Municipal de Saúde do Recife e não possuem valores em Reais definidos. 
Tabela 2. Alocação dos custos diretos sanitários segundo tipo de custos (período)

\begin{tabular}{|c|c|c|c|}
\hline \multicolumn{2}{|l|}{ Grupos de despesas } & \multirow{2}{*}{$\begin{array}{c}\text { Valor (R\$) } \\
978.096,10\end{array}$} & \multirow{2}{*}{$\begin{array}{c}\% \\
20,1\end{array}$} \\
\hline 1. Custo com pesso & & & \\
\hline Pessoal & $\begin{array}{l}\text { Profissionais de nível superior da assistência à saúde e administrativo, nível médio, } \\
\text { apoio e estagiários }\end{array}$ & $978.096,10$ & 20,1 \\
\hline \multicolumn{2}{|c|}{ 2. Custo de outras despesas (Overhead) } & $3.758 .573,02$ & 77,4 \\
\hline Material de consumo & & $74.428,78$ & 1,5 \\
\hline \multirow[t]{2}{*}{ Medicamentos } & Diabetes & $566.458,42$ & 11,7 \\
\hline & Cardiovasculares/hipertensão & $1.195 .966,00$ & 24,6 \\
\hline \multirow[t]{7}{*}{ Serviços de terceiros } & Administrativo & $489.223,34$ & 10,1 \\
\hline & Assistência ambulatorial & $10.845,08$ & 0,2 \\
\hline & Cursos, seminários, simpósios e congressos & $1.350,00$ & 0,0 \\
\hline & Assistência farmacêutica & 1.525 & 0,0 \\
\hline & Digitação e processamento de dados & $315.009,10$ & 6,5 \\
\hline & Equipamentos (manutenção e outras despesas) & $139.115,34$ & 2,9 \\
\hline & Locações & $39.569,96$ & 0,8 \\
\hline \multirow[t]{5}{*}{ Serviços básicos } & Água & $105.222,82$ & 2,2 \\
\hline & Energia elétrica & $412.141,84$ & 8,5 \\
\hline & Telefone & $135.324,61$ & 2,8 \\
\hline & Gás GLP & $4.947,96$ & 0,1 \\
\hline & Material de limpeza & $102.379,58$ & 2,1 \\
\hline \multirow[t]{2}{*}{ Suprimentos } & Compra de material & $43.200,00$ & 0,9 \\
\hline & Pagamento direto por serviços & $52.150,00$ & 1,1 \\
\hline \multirow[t]{6}{*}{ Outros } & Buffet, coffe-break, lanches & $12.274,34$ & 0,3 \\
\hline & Combustíveis & $4.229,00$ & 0,1 \\
\hline & Indenizações e restituições & $6.267,76$ & 0,1 \\
\hline & Refeições e gêneros alimentícios & $5.110,73$ & 0,1 \\
\hline & Uniformes & 237,27 & 0,0 \\
\hline & Ressarcimento de pessoal & $41.596,09$ & 0,9 \\
\hline \multicolumn{2}{|l|}{ 3. Custo de capital } & $118.623,00$ & 2,4 \\
\hline Edificações & Manutenção de bens e imóveis & $118.623,00$ & 2,4 \\
\hline \multicolumn{2}{|l|}{ Total } & $4.855 .292,12$ & 100 \\
\hline
\end{tabular}

Tabela 3. Composição dos custos totais e custo médio estimado de procedimentos ambulatoriais

\begin{tabular}{|c|c|c|c|c|c|c|}
\hline Procedimentos & $\begin{array}{c}\text { Custo de } \\
\text { funcionamento* }\end{array}$ & $\%$ & Custo de pessoal* & $\%$ & Custo total & Custo médio \\
\hline \multicolumn{7}{|l|}{ Consultas } \\
\hline Cardiologia & $127.546,95$ & 48 & $137.929,35$ & 52 & $265.476,30$ & 15,9 \\
\hline Endocrinologia & $138.479,54$ & 47,5 & $153.257,13$ & 52,5 & $291.736,67$ & 16,6 \\
\hline Nutrição & $11.479,23$ & 42,8 & $15.325,70$ & 57,2 & $26.804,93$ & 17,8 \\
\hline Oftalmologia & $273.314,89$ & 45 & $334.100,65$ & 55 & $607.415,54$ & 16,9 \\
\hline \multicolumn{7}{|c|}{ Exames cardiológicos } \\
\hline Ecocardiografia & $22.594,03$ & 24,4 & $69.886,86$ & 75,6 & $92.480,89$ & 31,2 \\
\hline Eletrocardiograma & $126.089,27$ & 80,4 & $30.651,40$ & 19,6 & $156.740,67$ & 9,4 \\
\hline Teste ergométrico & $12.025,86$ & 21,3 & $44.507,00$ & 78,7 & $56.532,86$ & 35,7 \\
\hline
\end{tabular}

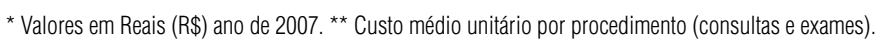


Tabela 4. Diferença entre o custo médio estimado em Reais $(\mathrm{R} \$)$ e valor reembolsado pelo SUS

\begin{tabular}{lccc}
\hline Procedimentos & $\begin{array}{c}\text { Custo médio* } \\
\text { (R\$) }\end{array}$ & $\begin{array}{c}\text { Valor SUS* } \\
\text { (R\$) }\end{array}$ & Diferença \% \\
\hline Consultas & 15,90 & 10,00 & +59 \\
Cardiologia & 16,60 & 10,00 & +66 \\
Endocrinologia & 17,80 & 6,00 & +197 \\
Nutrição & 16,90 & 10,00 & +69 \\
Oftalmologia & & & \\
Exames cardiológicos & 31,20 & 30,72 & +2 \\
\hline Ecocardiografia & 9,40 & 5,15 & +83 \\
Eletrocardiograma & 35,70 & 30,00 & +19 \\
\hline Teste ergométrico & & & \\
\hline
\end{tabular}

* Tabela SUS vigente em janeiro de 2008; valores em Reais (R\$).

\section{DISCUSSÃO}

Os custos com medicamentos para diabetes e doenças cardiovasculares representaram 36\% do total dos custos diretos sanitários no ano de 2007 em uma unidade de referência em Recife. Estudo realizado no México (20) avaliou a distribuição dos custos por tipo de serviço e concluiu que, para o manejo ambulatorial dos casos de diabetes e hipertensão arterial, os medicamentos representaram $56 \%$ e $43 \%$ dos custos de tratamento, respectivamente. No Brasil, um estudo em Pelotas (21) demonstrou que os medicamentos representaram cerca de $38 \%$ dos custos diretos no tratamento dessas doenças. Segundo uma estimativa do custo anual da hipertensão arterial, os medicamentos correspondem a $52,3 \%$ dos custos para o tratamento da doença no Sistema Único de Saúde (7). A participação do gasto do SUS com medicamentos aumentou de 5,4\% em 2002 para 10,7\% em 2007 (22).

Em relação à composição dos custos totais para o cálculo do custo médio unitário, o custo com profissionais apresentou maior peso para quase todos os procedimentos que tiveram seus valores unitários estimados (Tabela 3). Ao analisar os resultados do valor médio estimado dos procedimentos (Tabela 4), conclui-se que os valores de reembolso praticados pelo SUS cobrem apenas parte dos custos do serviço com pessoal. A composição dos custos totais pode servir como base para comparação do perfil de despesas dos serviços e da valoração do custo médio de procedimentos nos diferentes níveis de atenção.

As diferenças apresentadas entre o valor pago pelo SUS e o valor estimado (Tabela 4) contribuem para demonstrar o descompasso entre os valores pagos pelo SUS e os custos reais dos serviços, já verificados em ou- tros trabalhos. A tabela de procedimentos do SUS tem lógica de remuneração por nível de atenção. No nível hospitalar, os valores de ressarcimento das internações são pagos por meio de um pacote referente aos serviços profissionais e serviços hospitalares, em que estão inclú́dos os valores referentes a alimentação, taxas de sala, materiais hospitalares, medicamentos e exames de apoio diagnóstico; diferentemente do nível ambulatorial, em que os procedimentos são remunerados de forma linear segundo sua produção. Em ambos os níveis de atenção, os valores de reembolso têm se mostrado defasados em relação aos custos reais dos serviços (23-25).

Um estudo sobre os custos da insuficiência cardíaca apontou um custo unitário de R\$ 14,40 para consulta cardiológica e, no período do estudo, o reembolso do SUS só cobria $18 \%$ desse custo (23). Outro trabalho sobre financiamento da saúde no Brasil discutiu o subfinanciamento do SUS e demonstrou uma diferença de $+177,4 \%$ entre o valor real para a consulta em especialidade adulta e o valor pago pelo SUS, além de diferenças também expressivas para procedimentos de alta complexidade (24). Uma pesquisa em hospitais conveniados à rede pública demonstrou que o valor do desembolso do SUS para internações por pé diabético foi cerca de sete vezes inferior ao custo direto hospitalar (25). Esses aspectos reforçam a necessidade da criação de centros de custos para que a revisão da tabela SUS possa estar mais próxima da realidade, bem como a necessidade da participação tripartite no financiamento de procedimentos de média complexidade. Além disso, o baixo investimento na atenção básica e média complexidade pode impactar em consequências graves para o indivíduo e repercutir em altos custos para o SUS, como aponta estudo sobre impacto econômico de doenças cardiovasculares graves (14).

Quanto às limitações do estudo por se tratar de uma análise de custos, torna-se necessário ressaltar que os valores encontrados refletem a realidade da Unidade de Referência estudada. Além disso, os custos dos exames cardiológicos estão subestimados, pois, em razão da metodologia adotada, não foi possível incorporar os custos com a aquisição, a manutenção e a depreciação dos equipamentos e os custos com os materiais utilizados diretamente nesses exames.

É importante identificar os custos dos serviços de saúde, pois esse conhecimento permite conhecer os setores e as ações que precisam ser otimizados de forma a reduzir gastos, eliminar desperdícios com eficiência e preservar a qualidade do atendimento prestado (16). 
A implantação de um centro de custos torna possível contabilizar os recursos efetivamente utilizados em cada área de assistência dos serviços de saúde e, assim, aferir o valor real dos procedimentos. Em função da inexistência desse tipo de centro de custos, gerou-se uma estimativa do valor real. Consequentemente, os valores do custo médio dos procedimentos, apesar de apresentarem diferenças expressivas em relação aos valores da tabela do SUS, ainda podem estar subestimados, o que evidencia a necessidade de implantação de centro de custos nos serviços de saúde.

Do ponto de vista da eficiência da utilização dos recursos, os centros de custos nos serviços de saúde, quando bem implantados, geram uma visão abrangente dos recursos envolvidos na assistência, assim como a inexistência desses centros sugere alta possibilidade de ineficiência.

O presente estudo objetivou contribuir para a discussão sobre a importância das análises de custos das unidades de saúde, a fim de buscar a eficiência dos recursos utilizados na prestação dos serviços. Assim, os gestores da saúde poderão não apenas cuidar da racionalização e redução de custos, mas conscientizar-se da importância dessa análise na tomada de decisão, contribuindo para melhor alocação e priorização de recursos financeiros diante do cenário de escassez e com vistas também à melhor qualidade da atenção aos portadores de diabetes e hipertensão.

Declaração: os autores declaram não haver conflitos de interesse científico neste estudo.

\section{REFERÊNCIAS}

1. Cesse EAP, Freese E, Souza WV, Luna CF. Tendências da mortalidade por DCNT no Brasil: expansão ou redução?. In: Freese E, organizador. Epidemiologia, políticas e determinantes das doenças crônicas não transmissíveis no Brasil. Recife: Universitária da UFPE; 2006. p. 47-72.

2. Freese $E$, Fontbonne A. Transição epidemiológica comparada: modernidade, precariedade e vulnerabilidade. In: Freese E, organizador. Epidemiologia, políticas e determinantes das doenças crônicas não transmissíveis no Brasil. Recife: Universitária da UFPE; 2006. p. 17-46.

3. American Diabetes Association. Economic consequences of diabetes mellitus in the U.S. in 1997. Diabetes Care. 1998;21(2):296-309.

4. Georg AE, Duncan BB, Toscano CM, Schmidt MI, Mengue S, Duarte $C$, et al. Análise econômica de programa para rastreamento do diabetes mellitus no Brasil. Rev Saúde Pública. 2005;39(3):452-60.

5. Hogan P, Dall T, Nikolov P. American diabetes association. Economic costs of diabetes in the US in 2002. Diabetes Care. 2003;26 Suppl:917-32.

6. Williams R, Van Gaal L, Lucioni C. Assessing the impact of complications on the costs of type II diabetes mellitus. Diabetologia. 2002;45 Suppl 1:S13-7.
7. Dib MW, Riera R, Ferraz MB. Estimated annual cost of arterial hypertension treatment in Brazil. Rev Panam Salud Publica/Pan Am J Public Health. 2010;27(2):125-31.

8. Federação International de Diabetes. Economic impact of Diabetes. Disponível em: <http://www.diabetesatlas.org/content/background-papers-pdf> Acesso em: 18 de março de 2010.

9. Barceló A, Aedo C, Rajpathak S, Robles S. The cost of diabetes in Latin America and the Caribbean. Bulletin of the World Health Organization, Genebra. 2003;81(1):19-27.

10. Caporale JE, Calvo H, Gagliardino JJ. Costos de atención médica de personas con diabetes anteriores y posteriores a su hospitalización en Argentina. Rev Panam Salud Publica/Pan Am J Public Health. 2006;20(6):361-68.

11. González JC, Walker JH, EinarsonTR. Cost-of-illness study of type 2 diabetes mellitus in Colombia. Rev Panam Salud Publica/Pan Am J Public Health. 2009;26(1):55-63.

12. Arredondo A, Zuñiga $A$, Parada I. Health care costs and financial consequences of epidemiological changes in chronic diseases in Latin America: evidence from Mexico. Public Health. 2005;119:711-20.

13. Araújo DV, Tavares LR, Veríssimo R, Ferraz MB, Mesquita ET. Custo da insuficiência cardíaca no Sistema Único de Saúde. Arq Bras Cardiol. 2005;84(5):422-7.

14. Azambuja M, Foppa M, Maranhão MFC, Achutti AC. Impacto econômico dos casos de doença cardiovascular grave no Brasil: uma estimativa baseada em dados secundários. Arq Bras Cardiol. 2008;91(3):163-71.

15. Ribeiro RA, Mello RGB, Melchior R, Dill JC, Hohmann CB, Lucchese AM, et al. Custo anual do manejo da cardiopatia isquêmica crônica no Brasil. Perspectiva Pública e Privada. Arq Bras Cardiol. 2005;85(1):3-8.

16. Brasil. Ministério da Saúde; Secretaria-Executiva; Área de Economia da Saúde e Desenvolvimento. Avaliação econômica em saúde: desafios para gestão no Sistema Único de Saúde. Brasília: MS; 2008.

17. Drummond MF, Stoddart GL, Torrance GW. Métodos para la evaluación económica de los programas de atención de la salud. Madri: Diaz de Santos SA; 1991.

18. Brasil. Ministério da Saúde. Departamento de Informática do SUS. Sistema de Informações Ambulatoriais. Disponível em: http://siasih.datasus.gov.br/arqTabulacao.cfm. Acesso em: 9 mar. 2010.

19. Falk JA. Gestão de custos para hospitais: conceitos, metodologias e aplicações. São Paulo: Atlas; 2001.

20. Arredondo A, Damiám T. Costos económicos en la produción de servicios de salud: Del costo de los insumos al costo de manejo de caso. Salud Publica Mex. 1997;39(2):11-9.

21. Costa JSD, Fuchs SC, Olinto MTA, Gigante DP, Menezes AMB, Macedo $S$, et al. Cost-effectiveness of hypertension treatment: a population-based study. Sao Paulo Med J. 2002;120(4):100-4.

22. Vieira FS. Gasto do Ministério da Saúde com medicamentos: tendência dos programas de 2002 a 2007. Rev Saúde Pública. 2009;43(4):674-81.

23. Laurenti $R$, Buchalla CM, Carantin CVS. Doença isquêmica do coração. Internações, tempo de permanência e gastos: Brasil, 1993 a 1997. Arq Bras Cardiol. 2000;74(6):483-7.

24. Dain S. Os vários mundos do financiamento da saúde no Brasil: uma tentativa de integração. Cien Saude Colet. 2007;12 Suppl:1851-64.

25. Rezende KF, Nunes MAP, Melo NH, Malerbi D, Chacra AR, Ferraz $\mathrm{MB}$. Internações por pé diabético: comparação entre o custo direto estimado e o desembolso do SUS. Arq Bras Endrocrinol. Metab. 2008;52(3):523-30. 